\title{
Possibility of TAS-102 as an Early Line Therapy Against Advanced Colorectal Cancer
}

Taichi Yoshida, Koji Fukuda, Daiki Taguchi, Kazuhiro Shimazu, Masahiro Inoue and Hiroyuki Shibata*

Department of Clinical Oncology, Akita University, Graduate School of Medicine, Japan

\begin{abstract}
Introduction: New drugs have improved the outcome of metastatic colorectal cancer treatment. In Japan, more than 10 agents with at least seven different mechanisms such as 5-fluorouracil (5-FU), irinotecan (IRI), oxaliplatin, angiogenesis inhibitors, anti-epidermal growth factor receptor antibodies, regrafenib, and TAS-102, have been approved. With the increase in the number of alternatives, the importance of choosing the agents increases. To suppress the toxicities of agents is also necessary. To increase the quality of life of the patients should be taken into consideration for their longer survival. TAS-102 has proved to be an effective and safe agent for the 3rd or 4th lines of advanced colorectal cancer.
\end{abstract}

Presentation of case: We report a case of a patient who had suffered severe adverse events from standard firstline chemotherapy with 5-FU derivative and IRI. She did not want to undergo any more intensive chemotherapy. Toxicity and anxiety made her evade the standard combination chemotherapy. For this patient, TAS-102 achieved over $30 \%$ tumor shrinkage and more than 1-year progression-free survival with acceptable toxicities.

Conclusion: TAS-102 can result in a better outcome for a responder when given as an earlier line of therapy. In future, predictive biomarkers of TAS-102 should be identified for better use of this agent.

Keywords: TAS-102; Colorectal cancer; Early line; Predictive biomarker

\section{Introduction}

Colorectal cancer (CRC) is the third most common malignancy in men $(746,000$ cases, $10.0 \%$ of the total) and the second most common in women $(614,000$ cases, $9.2 \%$ of the total) worldwide [1]. The estimated mortality rates in both genders range from 20.3 per 100,000 men and 11.7 per 100,000 women in Central and Eastern Europe to 3.5 and 3.0, respectively, in Western Africa [1]. Almost a quarter of a century ago, metastatic CRC was treated using a single agent, 5-fluorouracil (5-FU), with a median survival time (MST) of approximately 1 year. Thereafter, relevant improvements in not only MST but also progression-free survival (PFS) and response rates have been achieved. The most recently reported MST has exceeded 30 months [2]. Almost a dozen agents, including 5-FU; its derivatives; Irinotecan (IRI); Oxaliplatin (OX); angiogenesis inhibitors, such as bevacizumab, ramucirumab, and aflibercept; anti-epidermal growth factor receptor antibodies, such as cetuximab and panitumumab; and multiple tyrosine kinase inhibitor regorafenib have contributed to this improvement. TAS-102, a new oral anti-cancer agent composed of a 1:0.5 mixture on a molar ratio of $\alpha, \alpha$, a-trifluorothymidine (TFD) and a thymidine phosphorylase inhibitor (TPI), tipiracil hydrochloride, has demonstrated improved results in MST and PFS, compared with a placebo, in a global phase 3 trial for metastatic CRC refractory to standard therapies [3]. A much safer profile of TAS-102 has also been confirmed, with grade $3 / 4$ neutropenia in $38 \%$ and febrile neutropenia in $4 \%$ patients. However, the response rate of TAS-102 was lower at $1.6 \%$. This might be due to its usage late in the line. Recently, we have observed a good response of TAS-102 for metastatic CRC as an early line of therapy.

\section{Case Presentation}

A 68-year-old female with rectal cancer underwent abdominoperineal resection at another hospital in January 2014. It was diagnosed as stage 1 , according to the TMN classification, and was histopathologically identified as an adenocarcinoma with mutant RAS (the mutation replaces the amino acid glycine with valine at position
12). In June 2014, she visited our department for chemotherapy because of multiple liver metastases detected by computed tomography (CT). Combined chemotherapy with S-1 and IRI (IRIS) was administrated in July 2014. It was interrupted because of severe abdominal pain, nausea, and vomiting on the 2 nd day. Her CT examination revealed toxic megacolon, and she was diagnosed with paralytic ileus (Figure 1a1d). She recovered after 3 weeks of conservative treatment. During this period, we checked the size of liver metastases by CT, which revealed tumor shrinkage (25\% reduction from baseline). Although IRIS was effective, she was afraid of receiving not only IRIS but also the idea of having chemotherapy itself. We proposed alternative methods of treatment, including surgery or radiofrequency ablation (RFA). She only agreed to receive 5-FU and l-leucovorin intravenous bolus therapy (FL). In September 2014, she was started on treatment with FL, given once a week for 6 consecutive weeks and 2 weeks off as one cycle. After one cycle, (almost 2 months), CT examination revealed a reduction of liver metastasis. In June, 2015, after completing 5 cycles of FL, regrowth of her liver metastases was confirmed. We proposed other treatment options, including liver surgery and RFA as well as more chemotherapy. However, she did not want any combination chemotherapies such as FOLFOX, FOLFIRI, angiogenesis inhibitors or regorafenib, because of their toxicities. She agreed with TAS- 102 because of its low toxicity profile. She started TAS-102 treatment in June 2015 at a $20 \%$ reduced dosage (from $50 \mathrm{mg}$ to $40 \mathrm{mg}$ ) twice a day. in consideration of her fear

*Corresponding author: Hiroyuki Shibata, Department of Clinical Oncology, Akita University, Graduate School of Medicine, Hondo 1-1-1, Akita, Japan, Tel: +81188846261; E-mail: hiroyuki@med.akita-u.ac.jp

Received: December 12, 2016; Accepted: December 25, 2016; Published: December 30, 2016

Citation: Yoshida T, Fukuda K, Taguchi D, Shimazu K, Inoue M, et al. (2016) Possibility of TAS-102 as an Early Line Therapy Against Advanced Colorectal Cancer. Oncol Cancer Case Rep 2: 119.

Copyright: $\odot 2016$ Yoshida T, et al. This is an open-access article distributed under the terms of the Creative Commons Attribution License, which permits unrestricted use, distribution, and reproduction in any medium, provided the original author and source are credited. 


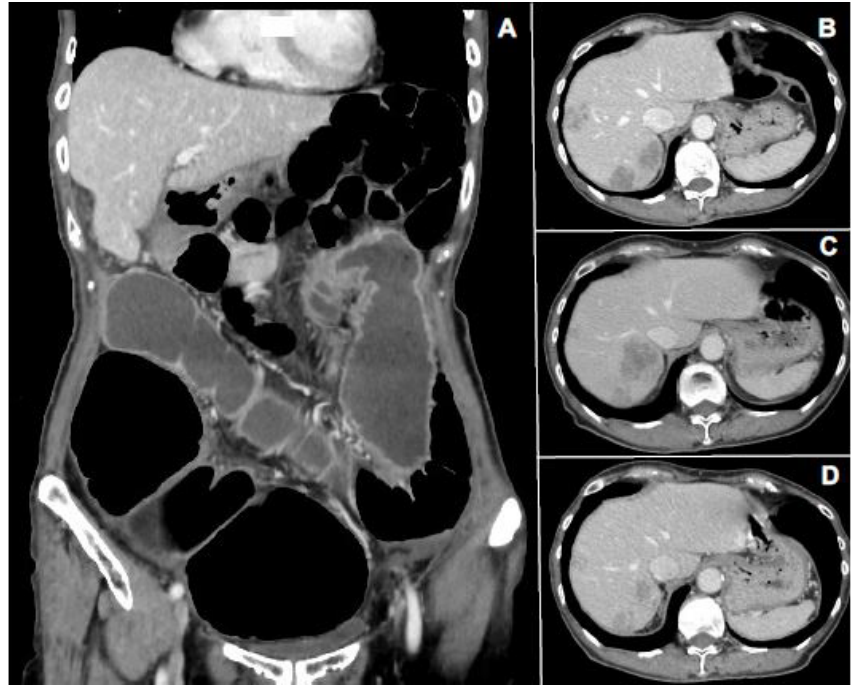

Figure 1: Clinical imaging during treatment, a) Toxic megacolon just after IR administration, b) Liver metastasis before FL treatment, c) Liver metastasis just before TAS-102 treatment, d) Maximum effect of liver metastasis with TAS-102.

of adverse reactions to the agents. Treatment was safely conducted with the occurrence of only grade 3 neutropenia, grade 1 nausea, constipation, and fatigue. Grade 3 neutropenia always delayed the start of a new cycle by 1 week. Three cycles later, CT revealed that the liver metastases had shrunk to $75 \%$ of baseline. Six cycles later, CT showed a $63 \%$ reduction from baseline (Figure 1d). Ten months from the start of TAS-102 administration, her liver metastases stayed within $67 \%$ of baseline, with no new lesions.

\section{Discussion}

Preclinical analysis indicates that TAS-102 can suppress the growth of 5-FU-resistant CRC cell line, DLD-1, inoculated in mice at a $73.2 \%$ frequency [4]. This data shows that TAS-102 has tumor shrinkage potential. The response rate (1.6\%) and PFS (2.1 months) of TAS-102 in the RECOURSE trial were lower [3]. Of course, this trial was conducted for heavily refractory cases and data include TAS-102 as late line therapy. Another trial for 5-FU and OX refractory cases as a second line indicates that the $95 \%$ confidential interval of PFS ranges from 1.9 to $6.2 \mathrm{months}$ with $50 \mathrm{mg} / \mathrm{m}^{2} /$ day to $60 \mathrm{mg} / \mathrm{m}^{2} /$ day TAS- 102 and $150 \mathrm{mg} /$ $\mathrm{m}^{2}$ IRI every 2 weeks [5]. It seems that even combined with IRI, TAS102 could not suppress tumor growth, compared with combination chemotherapies with 5-FU plus OX or 5-FU plus IRI. Second-line chemotherapy generally needs a much higher PFS, for example, that of 5-FU plus OX or 5-FU plus IRI was 8.2 months [6]. In our case, TAS-102 alone could shrink the size of 5-FU-resistant liver metastasis to approximately $60 \%$, and its PFS reached to over 12.5 months without severe toxicities. In this case, TAS-102 was very effective, and she was considered to be a responder. From this case it should be noted that if we could predict the response, TAS-102 is very beneficial in the earlier line for long-term disease control without severe adverse events. Therefore, it is very important to elucidate predictive biomarkers of TAS-102. Compared with 5-FU, TFD is incorporated into the DNA to a 300 -fold greater extent. Therefore, TAS-102 might be effective for 5-FU-resistant cases. Moreover, TFD inserted at T-sites is not excited by base excision repair proteins, such as uracil-DNA glycosylase, single-strand-specific monofunctional uracil DNA glycosylase, thymine-DNA glycosylase, or methyl-CpG-binding domain protein 4 [7,8]. Accordingly, these genes cannot be predictive biomarkers of TAS-102. A triphosphate form of TFD is incorporated by DNA polymerase a [9], and this enzyme can be a predictive biomarker. Data from a TAS-102-resistant cell line indicates that it has a normal thymidylate synthase activity but decreased thymidine kinase (TK) activity [10]. Another resistant cell line has an up-regulated secretory phospholipase A2 (sPLA2) expression [10]. Therefore, TK and sPLA2 might become biomarkers of TAS-102. TAS-102 is also composed of another compound, TPI, which has an angiogenesis inhibitory activity [11]. This compound can be studied as potential biomarker for metastatic CRC treatment. The predictive biomarkers for TAS-102 should be clarified to use this agent in the front line.

\section{Conclusion}

TAS-102 can be a safe and effective alternative for metastatic CRC as an early line of therapy, if the standard chemotherapy with combination of 5-FU plus OX or 5-FU plus IRI is toxic.

\section{Consent}

Informed consent was obtained from the patient of this report.

\section{Acknowledgement}

Hiroyuki Shibata received lecture fees from DAIICHI SANKYO Co., Ltd. The other authors declare that they have no conflict of interest.

\section{References}

1. http://glocan.iarc.fr/Pages/fact

2. Sargent $D(2015)$ Improved outcomes in metastatic colon cancer giving credit where credit is due. JAMA oncol 1:795-796.

3. Mayer RJ, Van Cutsem E, Falcone A, Yoshino T, Garcia-Carbonero R, et al (2015) RECOURSE study group. Randomized trial of TAS-102 for refractory metastatic colorectal cancer. N Engl J Med 372: 1909-1919.

4. Emura T, Suzuki N, Fujioka A, Ohshimo H, Fukushima M (2004) A nove combination antimetabolite, TAS-102, exhibits antitumor activity in FU-resistant human cancer cells through a mechanism involving FTD incorporation in DNA Int J Oncol 25: 571-578.

5. Doi T, Yoshino T, Fuse, N, Boku N, Yamazaki K, et al. (2015) Phase I study of TAS-102 and irinotecan combination therapy in Japanese patients with advanced colorectal cancer. Invest New Drugs 33: 1068-1077.

6. Bécouarn Y, Gamelin E, Coudert B, Négrier S, Pierga JY, et al. (2001) Randomized multicenter phase II study comparing a combination of fluorouracil and folinic acid and alternating irinotecan and oxaliplatin with oxaliplatin and irinotecan in fluorouracil-pretreated metastatic colorectal cancer patients. J Clin Oncol 19: 4195-4201.

7. Suzuki N, Emura T, Fukushima M (2011) Mode of action of trifluorothymidine (TFT) against DNA replication and repair enzymes. Int J Oncol 39: 263-270.

8. Matsuoka K, limori M, Niimi S, Tsukihara H, Watanabe S, et al. (2015) Trifluridine Induces p53-Dependent Sustained G2 Phase Arrest with Its Massive Misincorporation into DNA and Few DNA Strand Breaks. Mol Cancer Ther 14: 1004-1013.

9. Sakamoto K, Yokogawa T, Ueno H, Oguchi K, Kazuno H, et al. (2015) Crucial roles of thymidine kinase 1 and deoxy UTPase in incorporating the antineoplastic nucleosides trifluridine and 2'-deoxy-5-fluorouridine into DNA. Int J Oncol 46: 2327-2334.

10. Temmink OH, Bijnsdorp IV, Prins HJ, Oguchi K, Kazuno H, et al. (2010) Trifluorothymidine resistance is associated with decreased thymidine kinase and equilibrative nucleoside transporter expression or increased secretory phospholipase A2. Mol Cancer Ther 9: 1047-1057.

11. Suzuki N, Emura T, Fukushima M (2011) Mode of action of trifluorothymidine (TFT) against DNA replication and repair enzymes. Int J Oncol 39: 263-270. 